6

7

8

9

10

11 
2 The purpose of this study was to determine the effects of an eight-week plyometric training

3 intervention on measures of golf swing performance in highly skilled, adolescent golfers. Sixteen

4 male golfers were recruited to this study, being placed into two handicap and age-matched groups:

5 intervention and control. The intervention group completed an eight-week plyometric training

6 programme in addition to their golf-specific practice to study effects on clubhead speed (CHS), ball

7 carry distance $(B C D)$ and other associated measures. The control group continued to undertake their

8 golf-specific training with no plyometric training. The intervention group demonstrated significant

$9(p<0.05)$ improvements in CHS and BCD between pre and post trials. The control group showed no significant $(p>0.05)$ changes in golf performance. The results suggest that in highly skilled adolescent

11 golfers, eight-weeks of plyometric training may help to improve CHS and BCD by approximately $3 \%$.

12 However, large between participant performance differences were observed after the training

13 intervention. It was concluded that, for golfers wishing to improve their CHS and BCD, a golf-specific,

14 plyometric training programme could play an important part in the athlete's training programme.

KEY WORDS

Clubhead speed, ball carry distance, handicap, teenage athletes, adolescents, training. 
2 INTRODUCTION:

3 The achievement of consistent high performance in golf requires the player to have effective physical conditioning that will allow them to overcome the on-course demands encountered (Smith, 2010). Whilst there are many elements that comprise the golf game (putting, chipping, pitching, etc.), one ever-present demand is the ability to consistently hit full-swing shots (i.e. when driving, hitting approach irons, etc.), that are accurate and controlled, but that also promote maximum ball displacement (Burden, Grimshaw, \& Wallace, 1998). Recently, evidence has shown that professional golfers who drive the ball the furthest distance on the PGA Tour are significantly more likely to achieve lower scores on par-4 and par-5 holes (Hellström, 2014). If the desired outcome of a golfer's competitive round is to achieve the best possible 18 hole score, then it is reasonable to suggest that incorporating methods into their training which allows them to increase their maximal ball displacement, from the tee and from the fairway, would be of benefit.

Hume et al., (2005) suggested that one method by which golfers can improve their maximum ball displacement is through physical conditioning. Physical conditioning for golfers can also have the benefit of reducing the likelihood of injury and promoting faster recovery should injury occur (Grimshaw, et al., 2002). Physical conditioning for golf has also been shown to improve a combination of factors attributed to improving ball displacement, such as maximum $\mathrm{x}$-factor stretch (Bull and Bridge, 2012) and clubhead speed (CHS) (Lephart, et al., 2007). Furthermore, higher CHS is generally indicative of golfers with lower handicaps, regardless of age or training frequency (TorresRhonda, et al., 2011).

Previous studies associated with improving maximum ball displacement through physical conditioning have focused on various training modalities including traditional strength, "functional" training, flexibility, power, plyometric, and types of warm-up, typically of 8-12 weeks in duration in 
1

2

3

4

adult populations (Fletcher and Hartwell, 2004., Fradkin, Sherman, and Finch, 2004., Lamberth, et al., 2012., Lephart, et al., 2007). However, it has previously been observed that there has been less investigation into the role of physical conditioning in adolescent or college-aged players, primarily because of the difficulty of finding a group of suitable players (Torres-Rhonda, et al., 2011). Doan et al., (2006) and more recently, Bull and Bridge (2012) both looked at younger adults, but the mean age of the male participants in each study was over 18 years. Further, Bull and Bridge (2012) reported in detail on golf swing kinematics, but findings associated with CHS and Ball Carry Distance (BCD) were outside the scope of the study. With the participants in the study of Doan et al., (2006) not having official handicaps, they were estimated to be "zero" handicap. Those in the Bull and Bridge (2012) study were all category one ( $<5$ handicap) players. The participants in the study presented herein had an average age of less than 18 years and were all male.

On average the downswing phase of a drive in golf is around $230 \mathrm{~ms}$ (Hume, et al., 2005) which is considerably shorter than the duration needed to reach maximal force ( $>300 \mathrm{~ms}$ ) (Potteiger, et al., 1999). Therefore, it is important to train the explosive elements associated with the golf swing in order to be able to apply maximal force in the available time, increasing the rate at which force is produced. This is known as the rate of force development (RFD).

\section{Plyometric training}

Plyometric training is associated with increases in power production and RFD (Potteiger, et al., 1999). This form of resistance training emphasises the loading of the eccentric phase of a muscle action followed immediately by a concentric muscle action utilising the stretch shortening cycle (SSC) in an explosive manner (Vossen, et al., 2000). Often related to jumping and the use of the lower limbs, plyometric training has also been shown to increase upper body performance. Vossen et al., (2000) found that a six week plyometric push up training intervention increased performance significantly in a subsequent dynamic exercise measure over a standard push up control intervention in females. Limited studies have also observed beneficial increases in golf specific performance 
1

2

3

4

5

6

7

8

following plyometric interventions. Fletcher and Hartwell (2004) looked at the benefits of a combined strength and plyometric training programme on golf drive performance. Results suggested that an eight-week combined programme was sufficient to augment significant changes in CHS and drive distance in eleven male golfers (age $29 \pm 7.4$ years; Handicap $5.5 \pm 3.7$ ). Performance increases were attributed to muscular force increases and sequential body segment acceleration improvements, leading to greater swing velocities. This study however, utilised both heavy resistance and plyometric training methods, obscuring the potential of plyometric training alone for improving golf performance.

It has been proposed that the amount of force developed during the swing is related to the 'X-factor stretch' which is the difference observed between the rotation of the torso and the opposite rotation of the hips during transition into the downswing (Cheetham, et al., 2001). This leads to a delay in applied force, an increased stretch of the hip, trunk, and shoulder musculature resulting in a more rapid SSC (Chu, et al., 2010, Hume, et al., 2005) and a greater transfer of power from the larger body segments (legs) to the smaller distal body segments (arms) necessary for increases in force production (Knudson, 2007. Putnam, 1993). The active stretch seen during upper body, golf specific plyometric exercise may help to induce a greater stretch reflex and lead to subsequent performance increases relating to drive and swing performance (Fletcher and Hartwell, 2004). It seems that the use of golf specific strength and conditioning programs can lead to beneficial performance increases with regards to the swing and its characteristics. While there is support for the application of athletic conditioning programmes in male and female, recreational and elite, and adult and elderly golfers, little is known about the use in adolescent academy level golfers.

The aim of this study was to assess the effectiveness of an eight-week golf specific plyometric training intervention on subsequent swing performance in a group of highly-skilled adolescent golfers. It was hypothesised that the intervention group would significantly improve their outcome measures when compared to the control group. 
METHODS

Two groups (intervention and control) of participants were recruited from a local golf college.

5 Participants performed a pre- and post-training designed test protocol. The intervention group

6

7 completed the plyometric training programme (Table 1 ) in addition to their golf training (long and short game practice, practice rounds etc.) to ascertain whether this method of training, in this agedcohort, will have effects on factors such as CHS, BCD. The control group continued to perform golfspecific training and competition play whilst not performing plyometric training. Participants were evaluated in week one and week eleven. Weeks two to five consisted of the first training block. Week six was a rest week as the participants were on an academic break (half-term). Week seven to ten comprised the second training block. This is displayed graphically as Figure 1. Participants provided informed consent (parental/guardian consent for participants under 18 years) and completed medical questionnaires prior to joining the research study in accordance with the Declaration of Helsinki as revised in 2013. The protocols and procedures of the study were approved by the Institutional ethics committee.

\section{PARTICIPANTS:}

16 male golfers were recruited for the study. Golfers were placed into two handicap and age matched groups: intervention $(\mathrm{N}=8$, age $=17.3 \pm 1.5$ years, height $=1.73 \pm 0.09 \mathrm{~m}$, body mass $=68.0 \pm 7.6$ $\mathrm{kg}$, handicap $=4.7 \pm 3.0)$ and control $(\mathrm{N}=8$, age $=17.4 \pm 0.9$ years, height $=1.74 \pm 0.09 \mathrm{~m}$, body mass $=$ $74.3 \pm 10.8 \mathrm{~kg}$, handicap $=5.2 \pm 2.5)$. The study's participants all held official, competition handicap certificates and participated in golf training or match play at least three times per week. Participants were not involved in any other research programme. Participants in the intervention and control 
1

2

4

5

6

7

8

9

groups had limited resistance training experience ( $<$ six months) and no previous experience of plyometrics.

\section{PROCEDURES:}

\section{Training Intervention:}

The plyometric training programme was implemented as part of the golfers' winter and early spring preparation phase (January-March). The training programme consisted of two, four-week training blocks interspersed with a week rest period, enforced because of an academic holiday period at the golfers' college (Figure 1). The programme consisted of plyometric work i.e. jumping, bounding, medicine ball drills and rotational golf swing derivative exercises (Table 1). The intervention group completed the training programme in Table 1 twice per week under supervision from a qualified and experienced member of staff and trainee strength and conditioning students who were studying at undergraduate level. Each session was separated by a minimum of 24 hours. Sessions 1 and 2 were completed in the first four-week training block and sessions 3 and 4 in the second training block. A standardised warm up was used before each session. The warm up consisted of approximately 10 minutes mobilising and flexibility movements. These movements were intended to raise heart rate, increase blood flow to the working muscles, and to prepare the body for the upcoming programme by dynamically working relevant joints through a full range of motion. The warm up movements (leg swings, arm swings, light skipping drills etc.) were designed to closely replicate movement patterns that were to be performed in the main exercise programme. Participants were given instruction in the first session as to correct jumping and landing mechanics, and medicine ball throw technique. Participants were instructed to perform each repetition with maximum effort and as explosively as possible. Participant attendance at the sessions was monitored throughout the intervention. All participants attended all sessions.

\section{[Figure1 here]}


1 All movements performed were continuous in nature. For example, during the countermovement

2 jump drills, the participants completed successive jumps with no rest between each repetition to

3 make the drills more reactive in nature and promote a more rapid SSC. An outline of the exercise programme is shown in Table 1 . The medicine balls used in the programme are more accurately described as "slam balls". These medicine balls are filled with sand and can be "slammed" against floors and walls without rebounding. This allows the participant to perform the movements at maximum effort without having to catch the rebounding ball. This hard throwing effort and release of the ball is important for developing sequential acceleration of the hips, shoulders, elbows, and hands which mimics the golf swing action (Fletcher and Hartwell, 2004). The slam ball used had a mass of $5 \mathrm{~kg}$ with all other exercises being performed with bodyweight resistance only.

\section{Testing Protocol:}

All participants were assessed before and after the training intervention. After obtaining signed informed consent and a completed medical questionnaire the participants underwent standard anthropometric assessment prior to exercise. Height and body mass were measured with the participants wearing light shorts only. All testing was performed indoors in a laboratory setting. Participants completed a standardised warm up including dynamic stretching and practice swings not hitting a ball, followed by three practice shots, as has been utilised in previous research (Read et al., 2014). Participants performed three trials of the following physical assessments: Standing Vertical Jump (SVJ), Standing Broad Jump (SBJ), a Kneeling Chest Throw (KCT), and a Kneeling Rotational Throw (KRT) with a $5 \mathrm{~kg}$ medicine ball. Maximal countermovement jumps were used for the vertical jump assessment. An electronic contact jump mat was used to determine jump height (Just Jump, Probiotics, Huntsville, AL). Microswitches in the mat time the interval between take-off and landing. SBJs were measured as distance from toes at zero metres (take off position) and heels at landing. For the KCT, participants were required to kneel at zero metres with a $5 \mathrm{~kg}$ medicine ball held in both hands and in contact with the chest (as in the starting position for a chest pass). The 
1

2

3

4

participant then proceeded to throw the ball as far as possible using a pushing motion (elbow extension). The distance at which the ball landed was recorded. The KRT involved the participants kneeling in a lunge position before rotating and throwing the ball over their raised knee. Both set ups are shown in Figure 2. A kneeling position was used to limit the length of throw by the participants, owing to the size of the laboratory in which the tests were conducted.

Participants' golf swings were analysed using a golf simulator (P3ProSwing, Sports Vision Technologies, California, USA). According to manufacturer accuracy and peer-reviewed research the simulator monitors ball flight with a precision rating of $99 \%$ (Sommer and Ronnqvist, 2009). The simulator comprised a $22.9 \mathrm{~cm} \times 35.6 \mathrm{~cm}$ platform which was covered with a $1.5 \mathrm{~cm}$ high artificial grass top which was embedded into a larger artificial grass mat. The sensing platform contains 65 optical sensors positioned before and after the "impact zone". The golf ball was placed in the same position on the platform each time and the sensors measure direction (swing path e.g. in-to-out), speed, and angle of the clubhead (e.g. open, closed, square) immediately prior to and immediately after impact. The simulator then estimates distance and direction for each shot. The simulator produces 18 fields of data ranging from shot direction and club face angle, to heel/toe height. Whilst variables such as shot direction and angle of attack etc. are undoubtedly useful, it is outside the scope of this study to speculate as to if/how these may or may not be influenced by plyometric training. Because of the large amount of data generated, the results displayed within this study were delimited to the most pertinent variables based on the hypothesis of the study i.e. those outcome measures that are likely to be influenced by improvements in force generation as a result of plyometric training (Hit distance, CHS, ball speed, BCD, SVJ, SBJ, KCT, and KRT) . The testing set up is displayed in Figure 3.

[Figures 2, 3 here]

Participants were afforded three full practice swings off the artificial turf matting before transferring onto the sensing platform for recording. The participant then completed 10 full swings with a 5 -iron 
1 with approximately 45-60 seconds rest in between shots. The participants used their own 5-iron in

2 both assessments. Participants were asked to subjectively rate their shot on a scale of 1-5 with one

3 being very poor and five equalling a very good strike.

4 [TABLE 1 HERE]

5 Statistical Analyses:

6 Swing data was analysed post hoc for outliers using a box-and-whisker plot method. Upper and 7 lower boundaries were established using a multiple of 1.5 times the interquartile range, which was

8 added to or subtracted from the third and first quartiles, respectively. Any scores outside of the 9 upper and lower boundaries were omitted from the data. Swing data was also omitted if the 10 participant scored the shot as "very poor" on the aforementioned subjective scale, or if the 11 simulator did not successfully capture all of the data required.

12 A $2 \times 2$ mixed model repeated measures analysis of variance (ANOVA) was used to determine interactions between the independent variables: trial (within-participant pre and post) and group (between-participant, intervention or control), and the dependent variables: Hit distance, CHS, ball speed, BCD, SVJ, SBJ, KCT, and KRT. Pearson product moment correlations were used to assess relationships between handicap and $\mathrm{CHS}$, and handicap and percentage change in performance

17 between trials. Data was arranged and graphical images produced in Microsoft Excel (Microsoft 2010, Washington, USA) with statistical analysis computed using SPSS for Windows (v.20.0 IBM, New York, 1989-). Significance was set at an alpha level of $p<0.05$. Data is reported as a mean plus or minus the standard deviation.

21 Results: 
1 All participants attended all training sessions within the programme. A summary of results is shown

2 in Table 2. A total of 320 swings were collected and analysed. The outlier elimination process

3 outlined in the Methods section resulted in 15 shots being removed from the control group ( $N=4$ for "1"/poor strike, $\mathrm{N}=11$ outside $1.5 \mathrm{x}$ upper and lower boundaries) and 16 shots from the intervention group ( $\mathrm{N}=8$ for " 1 "/poor strike, $\mathrm{N}=8$ outside $1.5 \mathrm{x}$ upper and lower boundaries). The repeated measures ANOVA showed no significant differences between groups at baseline for hit distance, CHS, BCD, ball speed, SVJ, SBJ, KCT or KRT $(p>0.05)$. Post-hoc significant differences $(p<0.05)$ were observed pre-to-post-test (within-group) for both intervention and control in the SVJ, SBJ, KCT, and KRT trials. Between-group significant differences (Trial*Group interaction) were present for Hit Distance, CHS, BCD, Ball Speed, SVJ, SBJ, KCT, and KRT. The intervention group showed a mean preto-post difference in CHS of $3.9 \pm 3.0 \%$ whilst the control group differed by $-1.1 \pm 0.8 \%$ in CHS. BCD showed pre-post differences of $4.9 \pm 3.3 \%$ for the intervention and $-1.1 \pm 0.7 \%$ for the control. Mean coefficients of variation for swings conducted by the intervention group pre and post-test and the control group pre and post-test were $2.4 \%, 1.9 \%, 2.5 \%$ and $1.8 \%$, respectively. Figure 4 shows the relationship between mean CHS of the intervention and control groups in both trials. Figure 5 shows the change in CHS for each participant in study ordered from smallest change to largest change. Figure 6 displays a comparison in changes in CHS and handicap in the intervention group.

[FIGURES 4, 5, 6 HERE]

\section{DISCUSSION:}

This study found that hit distance, CHS, BCD, and ball speed significantly improved when using a 5iron following an eight-week plyometric training programme. The highly skilled adolescent golfers who underwent an eight-week plyometric training intervention showed a mean change in CHS of $4 \mathrm{~km} / \mathrm{h}$ (Figure 4). The study also showed a between-participant time*group significant difference in physical measures including SVJ, SBJ, and KRT. The control group were matched for age and handicap and showed no between-trials improvement in any measured golf swing characteristic. 
1

2

3

4

Recent literature suggests that athletic conditioning programmes based on flexibility, strength, and power can have a significant and beneficial effect on CHS and BCD (Bull and Bridge, 2012, Fletcher and Hartwell, 2004, Lephart, et al., 2007). The most similar study in the literature base to the present study is from Fletcher and Hartwell (2004). In their study, the authors assessed highly skilled adult golfers who were unfamiliar with plyometrics and demonstrated an improvement of $1.5 \%$ and 4.5\% in CHS and BCD respectively after eight-weeks of twice per week plyometric and strength training. The main differences between the study herein and the aforementioned paper were the collection and analysis methods to compute swing characteristics, the inclusion of a concurrent strength training programme, and the age of the participants. Despite the mean age of the participants in the Fletcher and Hartwell (2004) study being markedly different to those in this study, the improvements in CHS and BCD are similar. The present study did not incorporate strength training as part of the intervention in an effort to highlight the specific influence of plyometrics on golf swing performance characteristics. It would appear however, that both sets of golfers, adolescent and adult, could achieve beneficial adaptations to their CHS and BCD by utilising plyometric training.

Within the literature reviewed for this article, the largest improvement in BCD was observed in the study by Lephart et al., (2007). The authors reported a 7.7\% improvement in BCD between pre and post assessment trials when using a driver. Whilst the participants in the Lephart et al., (2007) study trained 3-4 times per week, the large improvement in their study when compared to the data in this example may be explainable by various means. Firstly, the authors did not utilise a control group in their study. When a control group is not used it is difficult to ascertain whether the improvements made are due to training induced changes or natural variation in golf swing performance (TorresRhonda, et al., 2011). The participants also used driver, the longest length golf club. It is known that driver swing speeds are the largest of any club. Therefore, any changes in performance will likely be amplified. Additionally, the golfers in the study were of a lower ability level (handicap $12.1 \pm 6.4$ ) when compared to the participants in this study $(4.7 \pm 3.0)$. It is known that as skill level decreases, 
1 variability in performance increases, particularly when performing a movement as complex and

2 intricate as the full golf swing (Meister, et al., 2011, Torres-Rhonda, et al., 2009). Betzler et al (2012)

3 have shown that, as handicap increases, so does the variability in club head speed. In the study,

4 golfers with handicaps 0-5 produced significantly faster clubhead speeds and with less variability

5 between shots, than those with handicaps above 6-12, 13-20, and 20+ (Betzler et al., 2012).

6 Therefore, the large improvements observed may have been masked by natural variability in

7 performance of the swing by less skilled participants. However, if the observations of the above

8 study are negated, then a combination of short duration, high volume, high intensity training such as

9 plyometric training may show improvements in BCD and CHS if monitored correctly to avoid

10 overtraining.

11 Recent evidence has shown that such "field-based" assessment methods such as those employed herein are reliable when correlating with CHS in single-figure handicap golfers (Read et al., 2013). Such assessment methods are useful for the strength and conditioning coach as they allow for accurate and efficient assessment of the physical capabilities of their golfers and highlight the effectiveness of a training intervention. While, non-rotational assessment methods such as the SVJ and SBJ might appear to have limited specificity to the golf swing, it has been shown in other sports with a rotational component, as well as golf, that there is coordinated sequencing of the kinetic chain from the leg musculature followed by trunk activation. This is also evident in the golf swing and it is possible that increased leg power, as inferred by jumping assessment, aids rapid RFD, influencing CHS (Read et al., 2013).

The study demonstrated that following eight weeks of plyometric training, the intervention group significantly increased their physical performance in the SVJ, SBJ, and KRT, when compared to the control group $(10.8 \%, 10.2 \%, 22.9 \%$, respectively). The control group however, also displayed significant differences $(3.0 \%, 3.2 \%, 3.5 \%$ respectively) between pre and post-tests in these variables, but this is likely explained by the typical error of these assessment methods. It has been shown that 
1 the between-session typical error of measure when expressed as a coefficient of variation

2 percentage for vertical jump and medicine ball assessment is around 4-6\% (Moir et al., 2008, Duncan

3 et al., 2008). Therefore, percentage improvements below this threshold could be interpreted as a

4 "learning effect" (Moir et al., 2008). The intervention group improved their SVJ and SBJ by over 10\%

5 and the KRT by over $20 \%$, which is clearly above the typical error of measure for these tests and

6 therefore, the authors are confident these represent true and meaningful changes.

7 Although there were improvements in CHS, BCD, jump, and throw data in the intervention group, it

8 is not possible to be certain that these changes occurred as a direct influence of the plyometric 9 programme as the underlying physiological mechanisms for the changes were not explored. The improvements in performance in the present study may have resulted from the intervention group undertaking an increased volume of training, irrespective of the plyometric training implemented. However, the balance of evidence from previous studies in the area suggests that plyometric training is likely to influence $\mathrm{CHS}$ and $\mathrm{BCD}$ to a greater extent than traditional high-repetition resistance training. It is thought that by manipulating the SSC through plyometric training that the athletic adaptations that occur will result in improved force-generating capacity (Fletcher and Hartwell, 2004). Speculatively, this could be due to increased RFD, however contact mats are unable to extract such data. Bull and Bridge (2012) showed that over an eight-week plyometric training programme, peak lead arm and lead hand speed did not alter in highly trained and skilled golfers who undertook no training (control), but improved significantly in the intervention group of a similar handicap. The age variance between the groups was three years ( $21.5 \pm 5.5$ and $24.4 \pm 8.8$ years), but the participants were adults whereas adolescents were studied herein. Previous studies that have used nonplyometric resistance training methods have reported much smaller improvements in CHS in adolescent athletes. Doan et al., (2006) reported only $0.6 \%$ improvements in CHS in male golfers of similar age and skill to the present study, having undertaken an 11-week programme. The programme undertaken by the participants in the Doan et al (2006) paper could be described as traditional resistance training. The high-repetition ranges (8-12) used in the study's programme 
1 design would likely influence muscular hypertrophy, rather than targeting improvements in rapid

2 SSC actions (Doan et al., 2006). This would suggest that the type of resistance training undertaken might influence the gains achieved by the athlete, particularly with reference to CHS.

4 To the authors' knowledge, no other research article commenting on golf swing performance and 5 physical conditioning has displayed individual participant adaptations to the training intervention as

6 displayed in this study as evidence by Figure 6. Previous studies have reported a correlation between handicap and CHS (Lindsay, et al., 2008., Meister, et al., 2011,) with low handicap golfers thought to generate more CHS than high handicap players. This phenomenon was not observed in this participant cohort, as there were large inter-individual responses to the plyometric training intervention (Figure 6). The lack of correlation between CHS and handicap may be explainable by the highly skilled nature of the participants within the study. The player with the highest handicap in the group was still a single figure handicap golfer and could therefore be considered as highly skilled. Secondly, as the participants in the intervention group in this study were all between the ages of 16 19 years, it is highly likely that they have not achieved full physical maturation. Because of this, the performers who are less well physically developed may have found other methods, excluding generating high $\mathrm{CHS}$ and $\mathrm{BCD}$, by which to achieve and maintain a low handicap (i.e. excelling in short game and putting). Indeed it has been stated in a review of elite golf that the greatest players on the PGA tour distinguish themselves from the rest of the players by possessing a more accurate short game (Hellström, 2008), although this has been recently disputed (Broadie, 2012). Therefore, it is possible to suggest that the highly skilled golfers in this study with lower CHS characteristics than expected may compensate by focussing more on other areas of their game to achieve and maintain a low-handicap.

In conclusion, it appears that in highly skilled adolescent golfers, a cohort group into which there has been little research, eight-weeks of plyometric training may help to improve performance characteristics such as $\mathrm{CHS}$ and $\mathrm{BCD}$ by around $3.9 \%$ and $4.9 \%$, respectively. However, large 
1 participant-participant differences were observed in improvement in CHS and BCD after the

2 plyometric training intervention. The underlying physiological mechanisms for these improvements

3 are unknown. For golfers wishing to improve their CHS and $B C D$, a golf-specific, plyometric training

4 programme could play an important part in their training programme.

5 PRACTICAL APPLICATIONS:

$6 \quad$ CHS and BCD can be improved over an eight-week period by undertaking two sessions per week of 7 plyometric training with skilled adolescent golfers. Skilled adolescent golfers with no resistance 8 training background can improve their CHS and BCD using a 5-iron, as well as jump and throw 9 performance through additional plyometric training added to their existing golf practice schedules. 10 Plyometric exercise offers the strength and conditioning practitioner a relatively safe, inexpensive, 11 and simple (when compared to more technically challenging explosive resistance training exercise 12 such as Olympic lifting movements and their derivatives) method by which to introduce athletic training movements to athletes who are of novice level with regards resistance training. As has been highlighted in this study however, there may be large inter-individual responses to this type of training stimulus. Taking a more individualised approach to the provision of a golf athlete's training provision, with increased frequency of performance monitoring, may help to induce $\mathrm{CHS}$ and $\mathrm{BCD}$

17 improvements.

REFERENCES:

Andersen, L. Aagaard, P. Influence of maximal muscle strength and intrinsic muscle contractile properties on contractile rate of force development. European Journal of Applied Physiology. 96(1); 46-52. 2006 and ball impact location on golfers' drives. Journal of Sports Sciences. 30(5); 439-448. 2012 
1

Bull, M. Bridge, M,W. The effect of an 8-week plyometric exercise program on golf swing kinematics. International Journal of Golf Science. 1, 42-53. 2012

Broadie, M. Assessing golfer performance on the PGA Tour. Interfaces. 42(2); 146-165

Burden, A. Grimshaw, P. Wallace, E. Hip and shoulder rotations during the golf swing of sub-10 handicap players. Journal of Sports Sciences. 16(2); 165-176. 1998

Cheetham, P. Martin, P. Mottram, R. St Laurent, B. The importance of stretching the "X-Factor" in the downswing of golf: The "X-Factor Stretch." Optimising performance in golf. 37(5); 192-199. 2001

Chu, Y. Sell, T. Lephart, S. The relationship between biomechanical variables and driving performance during the golf swing. Journal of Sports Sciences. 28(11); 1251-1259. 2010

Doan, B. Newton, R. Kwon, Y. Kraemer, W. Effects of physical conditioning on intercollegiate golfer performance. Journal of Strength and Conditioning Research. 20(1): 62-72. 2006

Duncan, M,J. Al-Nakeeb, Y. Nevill, A,M. Influence of familiarization on a backward, overhead medicine ball explosive power test. Research in Sports Medicine. 13(4): 345-352

Fletcher, I. Hartwell, M. Effect of an 8-week combined weights and plyometrics training program on golf drive performance. Journal of Strength and Conditioning Research. 18(1): 59-62. 2004

Fradkin, A,J. Sherman, C,A. Finch, C,F. Improving golf performance with a warm up conditioning programme. British Journal of Sports Medicine. 38: 762-765. 2004

Grimshaw, P. Giles, A. Tong, R. Grimmer, K. Low back and elbow injuries in golf. Sports Medicine. 32(10): 655-666. 2002

Hellström, J. Competitive elite golf: a review of the relationships between playing results. Technique and physique. Sports Medicine. 39(9): 723-741. 2008 
1 Hellström, J. Drive for dough: PGA tour golfers' tee shot functional accuracy, distance and hole score. Journal of Sports Sciences. 32(5): 465-9. 2014.

3

4

5

6

7

8

9

Hume, P. Keogh, J. Reid, D. The Role of Biomechanics in Maximising Distance and Accuracy of Golf Shots. Sports Medicine. 35 (5): 429-449. 2005

Knudson, D. Qualitative biomechanical principles for application in coaching. Sports Biomechanics. 6(1); 109-118. 2007

Lamberth, J. Hale, B. Knight, A. Boyd, J. Luczak, T. Effectiveness of a six-week strength and functional training program on golf performance. International Journal of Golf Science. 2. 33-42. 2013

Lennon, H.M. Physiological profiling and physical conditioning for elite golfers. Science and Golf III, Proceedings of the 1998 World Scientific Congress of Golf. A.J. Cochran, ed. St. Andrews, Scotland: Human Kinetics. 1998

Lephart, S. Smoliga, J. Myers, J. Sell, T. Tsai, Y. An eight-week golf-specific exercise program improves physical characteristics, swing mechanics, and golf performance in recreational golfers. Journal of Strength and Conditioning Research. 21(3): 860-869. 2007

Lindsay. D,M. Mantrop, S. Vandervoort, A, A. A review of biomechanical differences between golfers of varied skill levels. International Journal of Sports Science and Coaching. 3(supplement 1) 189-197. 2008

Meister. D,W. Ladd, A, L. Butler, E,E. Zhao, B. Rogers, A, P. Ray, C, J. Rose, J. Rotational biomechanics of the elite golf swing: benchmarks for amateurs. Journal of Applied Biomechanics. 27, 242-251. 2011

Moir, G. Shastri, P. Connaboy, C. Intersession reliability of vertical jump height in women and men. Journal of Strength and Conditioning Research. 22(6):1779-1784. 2008 
1 Potteiger, J. Lockwood, R. Haub, M. Dolezal, B. Almuzaini, K. Schroeder, J. Zebas, C. Muscle power

2 and fiber characteristics following 8 weeks of plyometric training. Journal of Strength and

3 Conditioning Research. 13(3); 275-279. 1999

4 Putnam, C. Sequential motions of body segments in striking and throwing skills: descriptions and 5 explanations. Journal of Biomechanics. 26(1): 125-35. 1993

6 Read, P,J. Miller, S,C. Turner, A,N. The effects of postactivation potentiation on golf club head 7 speed. Journal of Strength and Conditioning Research. 27(6): 1579-1582. 2013

8 Read, P,J. Lloyd, R,S. De Ste Croix, M. Oliver, J,L. Relationships between field-based measures of

9 strength and power and golf club head speed. Journal of Strength and Conditioning. 27(10): 2708-

$10 \quad 2713.2014$

11 Smith, F. The Role of Physiology in the Development of Golf Performance. Sports Medicine. $1240(8) ; 635-655.2010$

13 Sommer, M. Ronnqvist, L. Improved moto-timing: effects of synchronized metronome training on 14 golf shot accuracy. Journal of Sports Science and Medicine. 8: 648-656. 2009

15 Torres-Rhonda, L. Sanchez-Medina, L. Gonzalez-Badillo, J,J. Muscle strength and golf performance.

16 A critical review. Journal of Sports Science and Medicine. 10, 9-18. 2011

17 Vossen, J. Kramer, J. Burke, D. Vossen, D. Comparison of dynamic push-up training and plyometric 18 push-up training on upper- body power and strength. Journal of Strength and Conditioning 19 Research. 14(3): 248-253. 2000 
1 The authors would like to thank the golfers at the Royal Eastbourne Golf Academy for their time and

2 adherence to the training programme as well as their swing coaches and the academy manager for 3 promoting the athletic training programme.

4 